\title{
Confocal Microscopy System Performance: Foundations for Measurements, Quantitation and Deconvolution.
}

\author{
Robert M. Zucker and Tammy E. Stoker \\ Reproductive Toxicology Division, National Health and Environmental Effects \\ Research Laboratory, Office of Research Development, U.S. Environmental \\ Protection Agency, Research Triangle Park, North Carolina 27711
}

The confocal laser-scanning microscope (CLSM) has enormous potential in many biological fields. The reliability of the CLSM to obtain specific measurements and quantify fluorescence data is dependent on using a correctly aligned machine that contains a stable laser power. For many applications it is useful to know the CLSM system's performance prior to acquiring data images so the necessary resolution, sensitivity and precision can be obtained. Applications of deconvolution, FRET and quantification necessitate that the confocal is correctly configured and operating at the highest performance levels.

The most common method in many laboratories to measure system performance involves the use of a histological slide to create a "pretty picture". Although this test evaluates many parameters in a crude manner (laser power, field illumination and lateral resolution) that can influence a CLSM image, the interpretation of this histological image is subjective and range of acceptability is variable. In fact, many confocal microscopes can indeed obtain "pretty pictures" even when they are suboptimally functioning. Furthermore, it is impossible to compare similar machines for proper functionality when using only an image as a reference standard. This study involves methods that can be used to QA a CLSM. Without the use of these various performance tests, it cannot be absolutely determined whether CLSM machines are working at appropriate performance levels.

Tests methods have been devised on the Leica TCS-SP1 and TCS-4D confocal microscope systems to ensure that these machines are operating correctly. The tests measure the following: field illumination, lens functionality and lens clarity, field illumination, spectral registration, total laser power, laser stability, dichroic reflectance, spectral registration of the beams, axial resolution, scanning stability, overall machine stability, and system noise $(1,2)$. It is anticipated by using this type of test data, performance standards for confocal microscopes will be determined and the current subjectivity in evaluating CLSM performance will be eliminated. These tests will help serve as guidelines for other investigators to assess both the performance of their machines and the quality of data derived from their machines. These tests have been applied in a similar manner to Zeiss 510 confocal system.

One of our research objectives was to try to quantify fluorescence using a CLSM. We have used reproductive tissues (oocytes, embryos and fetal limbs) as model systems to quantify fluorescence. The distribution of cortical granules has been measured in normal and hormonally delayed rat zygotes. The pattern of fluorescence from these 
two oocyte groups suggests that extra sperm may enter an egg if there is incomplete release and uneven distribution of the cortical granule materials (illustrated below). We have used Bitplane (Imaris/Surpass) programs to quantify the amount of fluorescence at different gray scale values (GSV) in the zygotes with $3 \mathrm{D}$ visualization after using a water immersion $63 \mathrm{x}$ objective ( $\mathrm{Na} 1.2$ ). We have previously developed a technique that uses LysoTracker Red to measure late stage apoptosis in embryos (3). This dye accumulates in acidic regions associated with increased lysosomes and phagocytosis. Optimization of sample preparation techniques of fixation, $\mathrm{MEOH}$ dehydration and $\mathrm{BABB}$ clearing increased the transmission of laser light to enable the visualization of tissues as thick as $500 u M$ using a 10x objective $(\mathrm{NA}=0.40)$. We have used Bitplane software (Imaris/Surpass) to measure the number of particles and the volume of these particles at different gray scale values (GSV). This quantification technique will be discussed in terms of cell death using the following model systems: 1) somites in normal GD10 rat embryos; 2) GD14 developing limbs from dams exposed to different concentrations of 5-Flurouracil (5-FU); 3) GD 8-9 day developing embryos treated with different concentrations of toxicants and 4) cortical granule release in delayed zygotes.

\section{References}

1. Zucker, RM. Price OT. Cytometry 44:273-294 2001

2. Zucker, RM, Price OT. Cytometry 44: 295-308 2001

3. Zucker, RM. Hunter S. Rogers JM: Cytometry 33:348-354 1998

This abstract of a proposed presentation does not necessarily reflect EPA policy

A

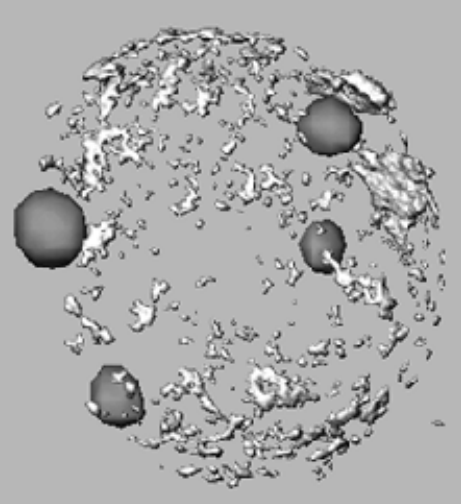

B

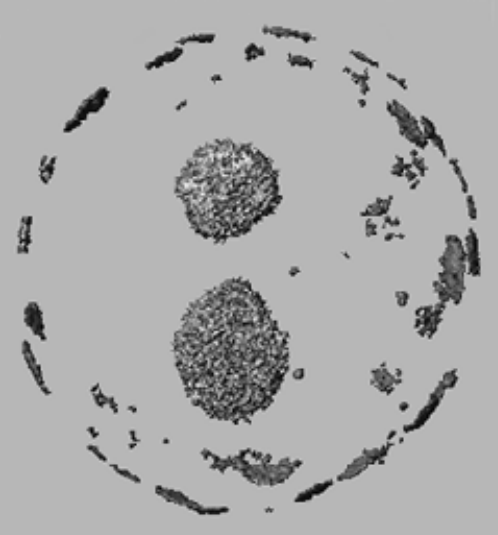

Polyspermic rat zygote (A,left) containing 3 sperm pronuclei and one-egg pronuclei stained with DAPI for the DNA and Lens Culinaris Aggluntin (LCA) Rhodamine labeled lectin for the cortical granules. The cortical granule distribution is uneven in this abnormal zygote. The light gray fluorescence represents cortical granules and can be quantified using Imaris/Surpass software. The normal zygote (B,right) contains one sperm pronuclei and one egg pronuclei. The cortical granule staining pattern is more symmetric and has less fluorescent intensity. 